\title{
Ablation of new onset atypical atrial flutter in a patient early after cardiac surgery
}

\author{
Cezary Maciejewski ${ }^{1, \mathrm{D}-\mathrm{F}}$, Paweł Czub ${ }^{2, \mathrm{D}-\mathrm{F}}$, Michał Peller ${ }^{1, \mathrm{D}-\mathrm{F}}$, Pawel Balsam ${ }^{1, \mathrm{~F}}$, Marcin Grabowski ${ }^{1, \mathrm{~F}}$, \\ Dominika Chojecka $^{1, \mathrm{D}}$, Dominika Żółcińska ${ }^{1, \mathrm{D}}$, Piotr Lodziński ${ }^{1, \mathrm{D}-\mathrm{F}}$ \\ A - Research concept and design, B - Collection and/or assembly of data, C - Data analysis and interpretation,
D - Writing the article, E - Critical revision of the article, F - Final approval of article
}

1 I Cardiology Clinic Medical University of Warsaw

2 Cardiac Surgery Clinic, Medical University of Warsaw

Address for correspondence:

Cezary Maciejewski, $1^{\text {st }}$ Cardiology Clinic, Medical University of Warsaw email: cmaciejewski6@gmail.com

Paweł Czub, Cardiac Surgery Clinic, Medical University of Warsaw email: pczub73@gmail.com

Michał Peller, $1^{\text {st }}$ Cardiology Clinic, Medical University of Warsaw email: michalpeller@gmail.com

Pawel Balsam, $1^{\text {st }}$ Cardiology Clinic, Medical University of Warsaw email: pawel.balsam@me.com

Marcin Grabowski, $1^{\text {st }}$ Cardiology Clinic, Medical University of Warsaw email: grabowski.marcin@me.com

Dominika Chojecka, $1^{\text {st }}$ Cardiology Clinic, Medical University of Warsaw email: dominikachojeckaa@gmail.com

Dominika Żółcińska, $1^{\text {st }}$ Cardiology Clinic, Medical University of Warsaw email: dominikazolcinska@interia.pl

Piotr Lodziński, $1^{\text {st }}$ Cardiology Clinic, Medical University of Warsaw email: piotr.lodzinski@me.com

Received: 2019-12-31

Revised:

Accepted: 2019-12-31

Final review:

DOI: $10.24255 / \mathrm{hbj} / 116027$

Key words:

ablation of atypical atrial flutter after cardiac surgery

\section{Abstract}

Atrial tachyarrhythmias are relatively common but mostly self-limiting complications after cardiac procedures. We report a case of atypical atrial flutter which developed early after surgery for severe mitral regurgitation and patent foramen ovale. The arrhythmia could not be managed by conservative measures and compromised the patient's recovery. The atypical atrial flutter was safely terminated by radiofrequency ablation despite the early post-operative period.

\section{Introduction}

The incidence of early post-cardiac surgery atrial tachyarrhythmias (excluding atrial fibrillation) is estimated at v17-35\% and arrhythmias are usually self-limiting and non-recurrent $t^{[1]}$. Right atrial incisional tachycardia is more frequently seen in patients with acquired heart disease while cavotricuspid isthmus-dependent atrial flutter is more frequently seen in individuals after surgery for congenital heart disease ${ }^{[2]}$. We present a case of atrial flutter (AFl) secondary to cardiac surgery for mitral valve regurgitation that was effectively managed by catheter ablation.

\section{Case report}

A 72-year-old man with hypertension and paroxysmal atrial fibrillation was admitted to the cardiac surgery department for planned surgery due to severe mitral valve regurgitation and patent foramen ovale with interatrial septal aneurysm that was detected 4 months earlier. Both defects resulted in substantial left-to-right leak. On admission the patient was in a good condition with sinus rhythm.

Cardiac surgery was performed: the anterolateral wall of the right atrium was incised anteriorly to the atrial appendage. Mitral valve P2 was excised, P1 and P2 sutured and implantation of the mitral annulus was performed (Physio 
Annuloplasty Ring Mitral $30 \mathrm{~mm}$ ) with an interatrial septal approach. The interatrial septum was sutured. During the postoperative period incessant atrial flutter with a variable conduction ratio occurred (ventricular rate 40-140 per minute). Multiple electrical cardioversions were performed with immediate $\mathrm{AFl}$ recurrence despite antiarrhythmic drugs. The rate control strategy using beta-adrenolytics and digoxin was also ineffective. From the clinical standpoint the persisting, non-controllable arrhythmia was the main problem that hindered recovery after the surgery in this patient. Despite the early post-operative period the ablation of arrhythmia substrate was planned.

Electrophysiological study revealed atypical right atrial flutter (cycle length $215 \mathrm{~ms}$ ) with 2:1 conduction. The right atrium was mapped with a PentaRay mapping catheter (CARTO Biosense Webster). Activation and voltage mapping and entrainment pacing maneuvers revealed the zone of decreased potentials at the anterolateral right atrium wall (Figure 1) and

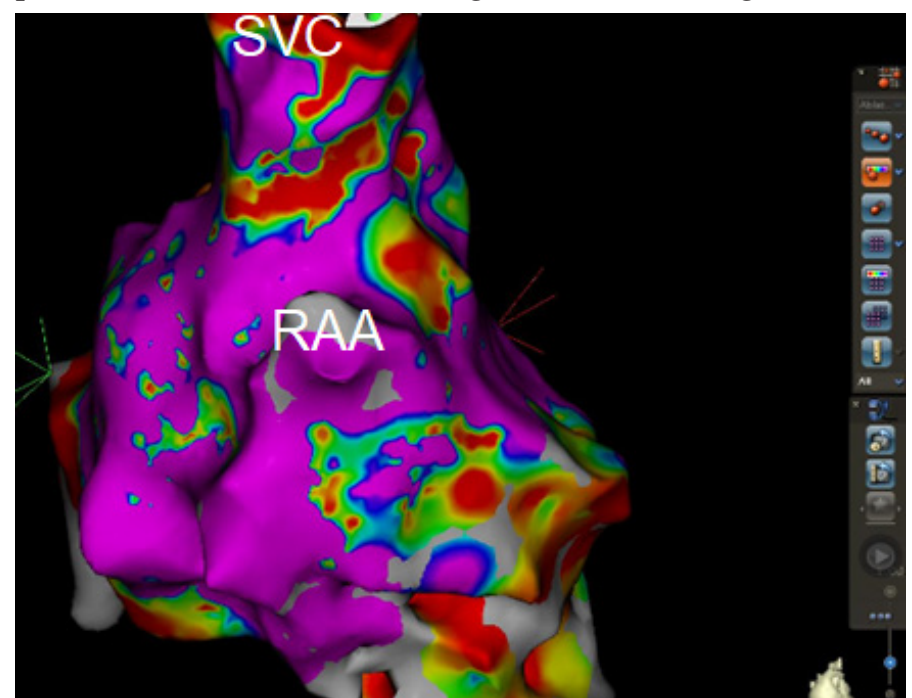

Figure 1. Potential, bipolar map; regions of decreased potentials anteriorly and superiorly to right atrial appendage (RAA) (anterolateral wall of the right atrium) are visible. SVC - superior vena cava

macroreentrant activation circulating around the right atrial appendage (Figure 2). The short ablation line - from the right

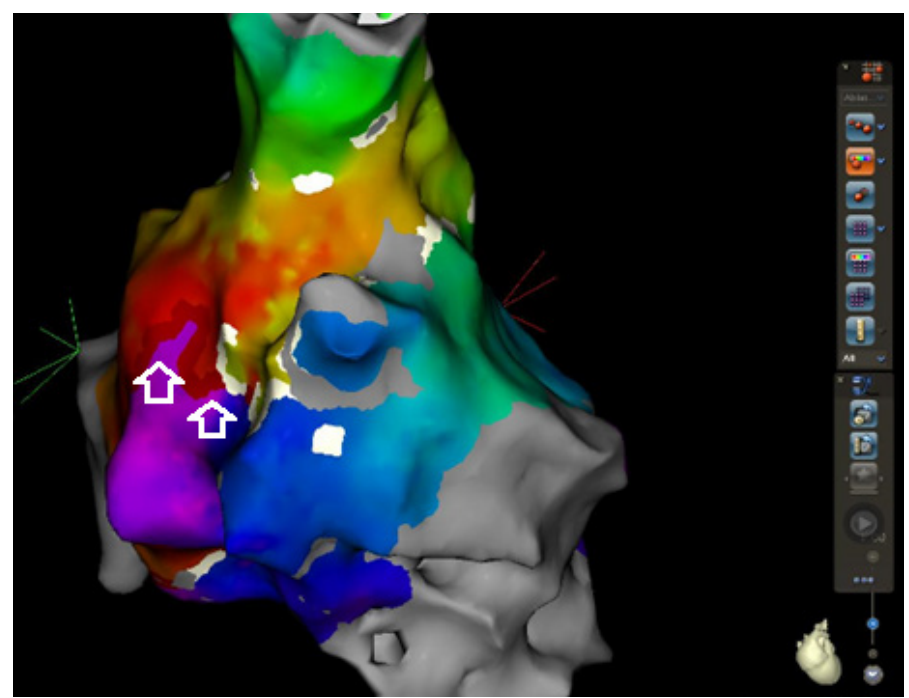

Figure 1. Local activation time (LAT) map of right atrium; macroreentrant activation circulating in a clockwise manner around the right atrial appendage. Early meets late activation zone marked with arrows. atrial appendage to the superior vena cava - was abandoned due to the risk of sinus node damage. A long line of radiofrequency applications between the right atrial appendage and inferior vena cava (Figure 3) was applied, resulting in arrhyth-

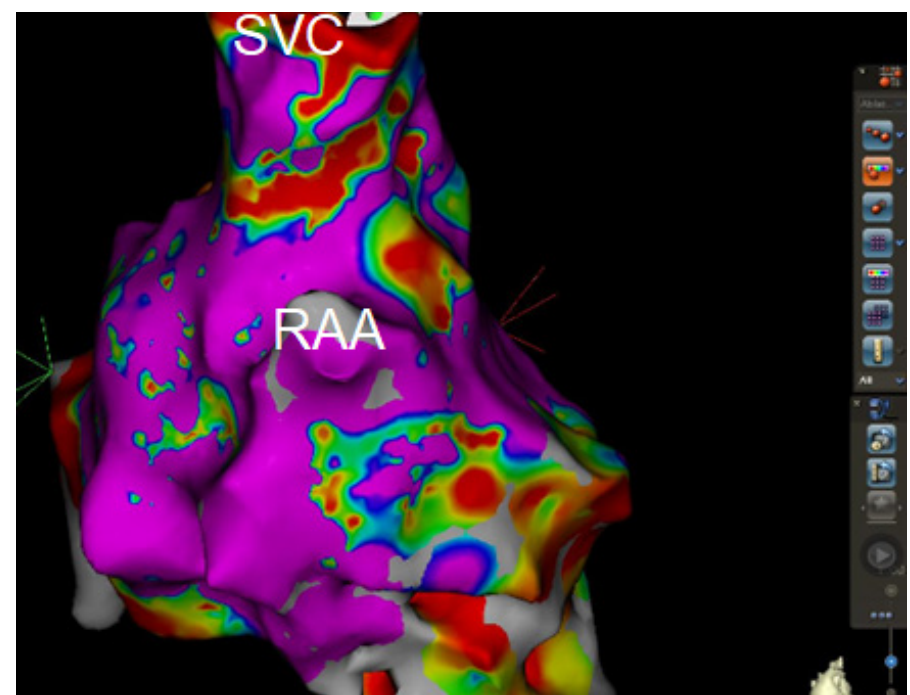

Figure 3. Ablation line from right atrial appendage to inferior vena cava that resulted in arrhythmia termination. View of lateral wall of the right atrium. Local activation time (LAT) map during pacing of superior vena cava - right atrium junction. Local activation revealed no conduction through the ablation line.

mia termination and restoration of sinus rhythm. Conduction block was confirmed with pacing maneuvers (Figure 3). The arrhythmia did not recur during the postoperative period and the patient was discharged home two weeks later.

\section{Discussion}

According to Marjaneh Fatemi et al. ${ }^{[3]}$, atrial flutter following non-congenital cardiac surgery is infrequent. After conversion to sinus rhythm the recurrence rate is relatively low $(12 \%)$ and therefore does not require invasive management - only $5 \%$ of patients require radiofrequency ablation of AFL in the early postoperative period. Surgical incisions applied to the atria create an anatomical substrate for arrhythmias. The central obstacle of macroreentry can be an anatomical structure (e.g. atrioventricular valves) or iatrogenic scar tissue (e.g. atriotomy). Right atrium incisional tachycardia is the most common non-cavotricuspid isthmus-dependent circuit and the likelihood of its occurrence increases with the extent of atriotomy ${ }^{[4]}$. The arrhythmia can be terminated by a linear lesion connecting the incision to another barrier: the inferior vena cava or tricuspid annulus ${ }^{[5]}$. This approach was used in the present case. As shown in our case, ablation is a safe and feasible approach for right-sided tachyarrhythmia induced by cardiac surgery that could not be managed by noninvasive strategies. 


\section{References}

1. Lukac, P., et al., Atrial incision affects the incidence of atrial tachycardia after mitral valve surgery. Ann Thorac Surg, 2006. 81(2): p. 509-13.

2. Tomita, Y., et al., Role of functional block extension in lesion-related atrial flutter. Circulation, 2001. 103(7): p. 1025-30.

3. Fatemi, M., et al., Atrial flutter after non-congenital cardiac surgery: Incidence, predictors and outcome. International Journal of Cardiology, 2011. 153(2): p. 196-201.

4. Pap, R., et al., Surgical technique and the mechanism of atrial tachycardia late after open heart surgery. J Interv Card Electrophysiol, 2012. 35(2): p. 127-35.

5. Kohári, M. and R. Pap, Atrial tachycardias occurring late after open heart surgery. Current cardiology reviews, 2015. 11(2): p. 134-140. al fibrillation. J Cardiol, 2019. 74(2): p. 150-155. 